\title{
Improved Expansion of Random Cayley Graphs
}

\author{
Po-Shen Loh"l and Leonard J. Schulman非 \\ ${ }^{1}$ Churchill College, University of Cambridge, Cambridge, CB3 ODS, UK \\ email: lpoealumni.caltech.edu \\ ${ }^{2}$ Department of Computer Science, California Institute of Technology, Pasadena, CA 91125, USA \\ email: schulman@caltech.edu
}

received Sep 2004, revised Dec 2004, accepted Dec 15, 2004.

\begin{abstract}
Alon and Roichman (1994) proved that for every $\varepsilon>0$ there is a finite $c(\varepsilon)$ such that for any sufficiently large group $G$, the expected value of the second largest (in absolute value) eigenvalue of the normalized adjacency matrix of the Cayley graph with respect to $c(\varepsilon) \log |G|$ random elements is less than $\varepsilon$. We reduce the number of elements to $c(\varepsilon) \log D(G)$ (for the same $c$ ), where $D(G)$ is the sum of the dimensions of the irreducible representations of $G$. In sufficiently non-abelian families of groups (as measured by these dimensions), $\log D(G)$ is asymptotically $(1 / 2) \log |G|$. As is well known, a small eigenvalue implies large graph expansion (and conversely); see Tanner (1984) and Alon and Milman (1984, 1985). For any specified eigenvalue or expansion, therefore, random Cayley graphs (of sufficiently non-abelian groups) require only half as many edges as was previously known.
\end{abstract}

Keywords: expander graphs, Cayley graphs, second eigenvalue, logarithmic generators

\section{Introduction}

All groups considered in this paper are finite.

Definition 1 Let $G$ be a group, and $S \subset G$ be a multiset. The Cayley graph $X(G, S)$ is the multigraph on vertex set $G$, with $n$ undirected edges connecting $g$ and $t g$ if t appears $n$ times in the multiset union $S \sqcup S^{-1}$, where $S^{-1}$ is the multiset $\left\{s^{-1}: s \in S\right\}$. The normalized adjacency matrix $A_{X(G, S)}^{*}$ is $1 /(2|S|)$ times the adjacency matrix of $X(G, S)$.

Definition 2 Let $M$ be an $n \times n$ matrix with real eigenvalues $x_{1}, \ldots, x_{n}$, where $\left|x_{1}\right| \geq \cdots \geq\left|x_{n}\right|$. Define $\lambda(M)=\left|x_{1}\right|$ and $\mu(M)=\left|x_{2}\right|$. Write $\mu(X(G, S))$ for $\mu\left(A_{X(G, S)}^{*}\right)$.

Definition 3 Let $D(G)$ be the sum of the dimensions of the irreducible representations of $G$.

\footnotetext{
${ }^{\dagger}$ Supported in part by the Marshall family, a Caltech Summer Undergraduate Research Fellowship, and an NSF REU supplement.

${ }^{\ddagger}$ Supported in part by NSF CAREER grant 0049092 and by a grant from the Okawa Foundation.

1365-8050 @ 2004 Discrete Mathematics and Theoretical Computer Science (DMTCS), Nancy, France
} 
Observe that $|G|^{1 / 2}<D(G) \leq|G|$. The upper bound is met only by abelian groups but is approached also by other groups whose irreducible representations are mostly low-dimensional, such as dihedral groups. The lower bound is approached, in the sense that $\log D(G) \rightarrow(1 / 2) \log |G|$, by a variety of families of groups possessing mostly high-dimensional irreducible representations.

Examples:

(a) The affine group $A_{p}$ over the prime field $G F(p) .\left|A_{p}\right|=p(p-1)$, while $D\left(A_{p}\right)=2 p-2$.

(b) The symmetric group $S_{n}$. $\left|S_{n}\right|=n$ !, hence $\log \left|S_{n}\right| \in n \log n-O(n)$, while $D\left(S_{n}\right) \in e^{O(\sqrt{n})} \sqrt{n !}$, hence $\log D\left(S_{n}\right) \in(1 / 2) n \log n+O(\sqrt{n})$.

(For the upper bound on $D\left(S_{n}\right)$, take the number of irreducible representations of $S_{n}$ times the maximum of their dimensions. The first of these is $p(n)$, the number of partitions of $n$, which has the asymptotic behavior $p(n) \sim \frac{1}{4 n \sqrt{3}} e^{\pi \sqrt{2 n / 3}}$. The second was shown by Vershik and Kerov (1985) to be bounded above by $e^{-k \sqrt{n}} \sqrt{n}$ ! for a positive constant $k$.)

Theorem 1 For any $\varepsilon>0$ the following holds for every sufficiently large group $G$. Let $S$ be a multiset of $c(\varepsilon) \log D(G)$ uniformly and independently sampled elements of $G$, for $c(\varepsilon)=4 e / \varepsilon^{2}$. Then we have $\mathrm{E}[\mu(X(G, S))]<(1+o(1)) \varepsilon$.

(Here and throughout $o(1)$ allows for a quantity tending to 0 for large $|G|$.) Russell and Landau (2004) have independently obtained a similar result.

As a detail note that in Alon and Roichman (1994), $S$ is generated by sampling without repetition (i.e., $S$ is a set), while we employ sampling with repetition. The principal benefit of this is to simplify the argument, but it also leads to some sharpening: the value of $c(\varepsilon)$ obtained in Alon and Roichman (1994) is slightly larger than given here, while substituting sampling with repetition into their argument leads to the same $c(\varepsilon)$.

\section{Proof}

The combinatorial outline of the proof follows that of Alon and Roichman; the heart of the improvement lies in taking a certain union bound over the irreducible representations, rather than over the entire regular representation, of the group.

\subsection{Decomposition into irreducible representations}

Fix a group $G$, and let $S$ be a multiset of $N$ elements of $G$. Let $T=S \sqcup S^{-1}$; let $\alpha$ be the element in the group algebra $\mathbb{C}[G]$ defined by:

$$
\alpha=\sum_{t \in T} \frac{1}{|T|} t
$$

Let the operator $L$ be the left-action of $\alpha$ on $\mathbb{C}[G]$. Its matrix representation with respect to the standard basis is the normalized adjacency matrix of $X(G, S)$. The Fourier Transform $\mathcal{F}$ is an algebra isomorphism from $\mathbb{C}[G]$ to $\bigoplus_{r=1}^{R} \mathcal{M}_{r}$, where $R$ is the number of irreducible representations of $G$, and $\mathcal{M}_{r}=\operatorname{Mat}_{d_{r} \times d_{r}}(\mathbb{C})$. Hence the eigenvalues of $L$ are the same as the eigenvalues of the left-action of $\mathcal{F}(\alpha)$ on $\oplus \mathcal{M}_{r}$. Explicitly, 


$$
\mathcal{F}(\alpha)=\bigoplus_{r=1}^{R}\left(\sum_{t \in T} \frac{1}{|T|} \rho_{r}(t)\right),
$$

where $\rho_{r}: G \rightarrow \mathcal{M}_{r}$ are the (unitary) irreducible representations, expressed with respect to fixed bases. Focus on an arbitrary component $r$ of $\mathcal{F}(\alpha)$ : let $\Psi_{r}=(1 /|T|) \sum_{t \in T} \rho_{r}(t)$.

Since $\Psi_{r}$ is an average of unitary matrices, its eigenvalues are bounded in absolute value by 1 .

Let $\rho_{1}$ be the one-dimensional trivial representation $\rho_{1}: G \mapsto \mathbb{C}$. Then for any $S, \Psi_{1}=1$. Therefore, $\mu(X(G, S))=\lambda(A)$, where $A$ is the following block-diagonal matrix:

$$
A=\left(\begin{array}{cccc}
\Psi_{2} & 0 & \ldots & 0 \\
0 & \Psi_{3} & \ldots & 0 \\
. & . & \ldots & . \\
0 & 0 & \ldots & \Psi_{R}
\end{array}\right)
$$

\subsection{From eigenvalues to random walks}

Fact 1 Let $M$ be a square matrix with real eigenvalues. Then for every positive integer $m$,

$$
\lambda(M) \leq\left(\operatorname{Tr}\left(M^{2 m}\right)\right)^{1 / 2 m} .
$$

Because of the symmetric construction of $T, A$ is Hermitian. By convexity,

$$
\mathrm{E}[\mu(X(G, S))] \leq\left(\mathrm{E}\left[\operatorname{Tr}\left(A^{2 m}\right)\right]\right)^{1 / 2 m} .
$$

Since $A$ is block-diagonal, $A^{2 m}$ shares the same block structure, with blocks $\Psi_{i}^{2 m}(2 \leq i \leq R)$.

$$
\begin{aligned}
\operatorname{Tr}\left(A^{2 m}\right) & =\sum_{r=2}^{R} \operatorname{Tr}\left(\Psi_{r}^{2 m}\right) \\
& =\sum_{r=2}^{R}\left(\sum_{t_{1}, \ldots, t_{2 m} \in T} \frac{\chi_{r}\left(t_{1} \cdots t_{2 m}\right)}{|T|^{2 m}}\right) \\
& =\sum_{r=2}^{R} \sum_{g \in G} \chi_{r}(g) \frac{N_{g}}{|T|^{2 m}},
\end{aligned}
$$

where $\chi_{r}$ is the character of $\rho_{r}$ and $N_{g}$ is the number of ways to produce $g$ as a product of $2 m$ (not necessarily distinct) elements of $T$.

Definition 4 Let $\boldsymbol{R} \boldsymbol{W}$ denote the following random walk process.

(1) Choose a uniform random word of length $2 m$ from the free monoid on the $N$ letters $\left\{a_{1}, a_{2}, \ldots, a_{N}\right\}$ (e.g., $\left.a_{2} a_{5} a_{5}^{-1} a_{1}^{-1} a_{7} a_{3}\right)$.

(2) Reduce the word in the free group (e.g., $\left.a_{2} a_{5} a_{5}^{-1} a_{1}^{-1} a_{7} a_{3} \rightarrow a_{2} a_{1}^{-1} a_{7} a_{3}\right)$. 
(3) Uniformly and independently assign (not necessarily distinct) group elements to the letters that appear in the remaining word, and evaluate the product in $G$.

Let $\mathbf{R W}_{g}$ be the event that the result is $g . \operatorname{Pr}\left(\mathbf{R} \mathbf{W}_{g}\right)=N_{g} /|T|^{2 m}$, so

$$
\mathrm{E}\left[\operatorname{Tr}\left(A^{2 m}\right)\right]=\sum_{g \in G} \operatorname{Pr}\left(\mathbf{R W}_{g}\right) \sum_{r=2}^{R} \operatorname{Re} \chi_{r}(g)
$$

\subsection{Mixing in the random walk}

Definition 5 Let $\omega$ be a reduced word as obtained via step (2) of process $\mathbf{R W}$ (definition (4). Say that $\omega$ has a singleton if there is an $i$ such that the number of occurrences of $a_{i}$ in $\omega$ plus the number of occurrences of $a_{i}^{-1}$ in $\omega$ is exactly one.

Let $\Omega$ be the event that the reduced word has a singleton. Now:

$$
\begin{aligned}
& \sum_{g \in G} \operatorname{Pr}\left(\mathbf{R} \mathbf{W}_{g}\right) \sum_{r=2}^{R} \operatorname{Re} \chi_{r}(g) \\
= & \sum_{g \in G} \operatorname{Pr}\left(\Omega \wedge \mathbf{R W}_{g}\right) \sum_{r=2}^{R} \operatorname{Re} \chi_{r}(g)+\sum_{g \in G} \operatorname{Pr}\left(\bar{\Omega} \wedge \mathbf{R} \mathbf{W}_{g}\right) \sum_{r=2}^{R} \operatorname{Re} \chi_{r}(g) \\
\leq & \sum_{g \in G} \operatorname{Pr}\left(\Omega \wedge \mathbf{R W}_{g}\right) \sum_{r=2}^{R} \operatorname{Re} \chi_{r}(g)+\operatorname{Pr}(\bar{\Omega}) D(G) .
\end{aligned}
$$

Lemma $1 \operatorname{Pr}\left(\boldsymbol{R} \boldsymbol{W}_{g} \mid \Omega\right)=1 /|G|$.

Proof: In step (3) of RW (definition 4), assign the singleton element last; then, there will exist a unique group element that makes $\omega$ evaluate to $g$.

Comment: This lemma replaces an upper bound of $1 /|G|+O\left(m / G^{2}\right)$ in Alon and Roichman (1994), the additional term being the result of their requiring distinct assigments in step (3). This additional term leads in turn to an extra summand of $e^{-b}$ in the analogue, in their work, of the center expression in Inequality [2].

By Lemma 1 and the orthogonality of characters, the first term of Bound (1) vanishes. Combining our inequalities:

$$
\mathrm{E}[\boldsymbol{\mu}(X(G, S))] \leq\left(\mathrm{E}\left[\operatorname{Tr}\left(A^{2 m}\right)\right]\right)^{1 / 2 m} \leq \operatorname{Pr}(\bar{\Omega})^{1 / 2 m} D(G)^{1 / 2 m} .
$$

To bound $\operatorname{Pr}(\bar{\Omega})$, we follow the spirit of Alon and Roichman (1994) and define the following two events in terms of the quantity $M=2 m(1-\log \log 2 m / \log 2 m)$ :

(A) After step 22) of RW (definition 4, the length of the reduced word is less than $M$.

(B) After step (2) of $\mathbf{R W}$ (definition 4), the length of the reduced word is at least $M$, but there are no singletons. 
Clearly, $\operatorname{Pr}(\bar{\Omega}) \leq \operatorname{Pr}(A)+\operatorname{Pr}(B)$. Alon and Roichman (1994) produced these bounds:

$$
\begin{aligned}
& \operatorname{Pr}(A) \leq 2^{2 m}(2 / N)^{m \log \log 2 m / \log 2 m} \\
& \operatorname{Pr}(B) \leq 2^{M}(m / N)^{M / 2}
\end{aligned}
$$

Substituting $N=c(\varepsilon) \log D(G)$ and $2 m=(1 / b) \log D(G)$, for any constant $b$, we obtain an expression almost identical to one of Alon and Roichman (1994), except that $|G|$ 's are replaced by $D(G)$ 's:

$$
\operatorname{Pr}(\bar{\Omega})^{1 / 2 m} D(G)^{1 / 2 m} \leq(1+o(1)) e^{b} \sqrt{\frac{2}{b c(\varepsilon)}} \leq(1+o(1)) \varepsilon
$$

where we use the choices $c(\varepsilon)=4 e / \varepsilon^{2}$ and $b=1 / 2$. 


\section{References}

N. Alon and V. D. Milman. Eigenvalues, expanders and superconcentrators. In Proc. 25th IEEE FOCS, pages 320-322, 1984.

N. Alon and V. D. Milman. $\lambda_{1}$, isoperimetric inequalities for graphs and superconcentrators. J. Comb. Theory, Series $B, 38: 73-88,1985$.

N. Alon and Y. Roichman. Random cayley graphs and expanders. Random Structures and Algorithms, 5: 271-284, 1994.

A. Lubotzky. Cayley graphs: Eigenvalues, expanders and random walks. In P. Rowlinson, editor, Surveys in Combinatorics 1995, pages 155-189. Cambridge University Press, 1995.

A. Lubotzky, R. Phillips, and P. Sarnak. Ramanujan graphs. Combinatorica, 8:261-277, 1988.

A. Russell and Z. Landau. Random Cayley graphs are expanders: a simple proof of the Alon-Roichman theorem. Electronic Journal of Combinatorics, 11(1):R62, 2004.

R. M. Tanner. Explicit construction of concentrators from generalized n-gons. SIAM J. Alg. Disc. Meth., 5:287-293, 1984.

A. Vershik and S. Kerov. Asymptotics of the largest and the typical dimensions of irreducible representations of a symmetric group. Funktsional. Anal. i Prilozhen, 19(1):25-36, 1985. English translation: Functional Analysis and its Applications, 19(1):21-31, 1985. 